1.04-2.41); and cocaine use on more than 10 occasions $(\mathrm{OR}=1.98$, 95\% CI: 1.31-2.99).

Conclusions The results demonstrated that construction interventions should be developed to address preventable risk factors. Young construction workers could benefit not only from enhanced work-place injury preventions, but also health behaviour interventions.

\section{TRANSITIONS BETWEEN UPPER EXTREMITY MUSCULOSKELETAL SYMPTOMS AND WORK LIMITATION OUTCOMES: A PROSPECTIVE STUDY}

'Bethany Gardner, ${ }^{1}$ Ann Marie Dale, ${ }^{2}$ Alexis Descatha, 'Bradley Evanoff. 'Washington University in St. Louis, St. Louis, MO, USA; ${ }^{2}$ Université Versailles-Saint Quentin, Versailles, France

\subsection{6/oemed-2014-102362.135}

Objectives Selection of appropriate outcome measures in studies of work-related musculoskeletal disorders (MSDs) directly affects the observed exposure-response relationship. Considering that many different factors might affect different stages of disease severity, we examined disability outcomes that represent a spectrum of disease in a newly hired working population and described the transitions between various states of symptoms and disability. Method From July 2004 to October 2006, 1107 newly hired workers were recruited to participate in the study. Subjects completed self-reported questionnaires including demographics, medical and work history, and current symptom and work status, nerve conduction studies, and a physical exam. Surveys were repeated at 6,18 , and 36 month follow-up; 827 subjects $(75 \%)$ completed all follow-ups and were included in the analysis. The outcomes of interest were presence of upper extremity symptoms and limitations in work abilities, productivity, job restrictions, lost time, and job changes due to these symptoms.

Results A majority of workers (72\%) reported symptoms at least once during the study, yet less than half $(44-46 \%)$ reported symptoms within any single follow-up period. Similarly, 31\% of workers reported work limitations due to their symptoms at least once during the study, but only $15-16 \%$ within any single follow-up period.

Conclusions These results provide evidence for the dynamic nature of both MSD symptoms and work abilities over time, which has been theorised but with few explicit studies. If the risk factors for these outcomes differ, this may explain some of the lack of clarity in the current literature on work-related risk factors and MSD.

\section{THE LIFETIME RISK APPROACH TO ESTIMATING THE BURDEN OF OCCUPATIONAL CANCER}

${ }^{1}$ Lin Fritschi, 'Renee Carey, 'Susan Peters, ${ }^{1}$ Alison Reid, ${ }^{2}$ Tim Driscoll, ${ }^{3}$ Lesley Rushton, ${ }^{4}$ Deborah Glass, ${ }^{3}$ Sally Hutchings. 'The University of Western Australia, Perth, Australia; ${ }^{2}$ Sydney University, Sydney, Australia; ${ }^{3} /$ mperial College, London, UK; ${ }^{4}$ Monash University, Melbourne, Australia

\subsection{6/oemed-2014-102362.136}

Objectives The main approaches to estimating the burden of occupational cancer are attributable risk and lifetime risk. In this presentation we will explain why we used the lifetime risk approach.

Method The lifetime risk of cancer is an estimation of an individual's risk of being diagnosed with cancer during their life (without considering occupational exposures). The lifetime risk for the general population $\left(\mathrm{LR}_{\mathrm{GP}}\right)$ is estimated by multiplying cohort person-years-at-risk (from life table data) by age-sex specific incidence rates.

The excess lifetime risk of cancer in a cohort of workers exposed to the carcinogen of interest ( $\left.\mathrm{LR}_{\text {exposed }}\right)$ is a product of the $\mathrm{LR}_{\mathrm{GP}}$ and the excess relative risk of developing cancer associated with that exposure. $\mathrm{LR}_{\text {exposed }}$ is multiplied by the prevalence of exposure to obtain the number of cancers attributable to the exposure in the general working population.

Results The lifetime risk approach estimates the number of cancers which would occur over a number of years in the future, due to exposures in a specific year. In contrast, the attributable risk approach estimates the number of cancers which would occur in a specific year due to exposures over a number of years in the past. Because we had exposure prevalence information for a specific year based on a national survey, we determined that the lifetime risk approach was more applicable in our case.

Conclusions The lifetime risk approach is an alternative method for calculating burden of disease when exposure prevalence information is available.

\section{OBTAINING POPULATION ESTIMATES OF THE PREVALENCE OF OCCUPATIONAL EXPOSURE}

${ }^{1}$ Lin Fritschi, ${ }^{1}$ Renee Carey, 'Susan Peters, 'Alison Reid, ${ }^{2}$ Deborah Glass, ${ }^{2}$ Geza Benke, ${ }^{3}$ Tim Driscoll. ${ }^{1}$ The University of Western Australia, Perth, Australia; ${ }^{2}$ Monash University, Melbourne, Australia; ${ }^{3}$ Sydney University, Sydney, Australia

\subsection{6/oemed-2014-102362.137}

Objectives Good occupational health policy requires an overall understanding of the proportion of the working population who are exposed to hazards at work. This is difficult to estimate when nearly three-quarters of the workforce are in small and medium sized companies and so not easily surveyed or monitored. We are undertaking a series of national surveys of the workforce to estimate how many people are exposed to hazards, where those people work, and to identify areas where controls could be used more effectively.

Method A random sample of the working population were invited to participate in a telephone interview regarding carcinogens at work using a web-based application (OccIDEAS). Participants were asked about their job tasks and predefined algorithms were used to automatically assign exposures.

Results Overall, $40.3 \%$ of the working population were estimated to be exposed to at least one of the 38 carcinogens we were interested in. Farmers, heavy vehicle drivers and miners were the most likely to be exposed. The most common exposures were solar radiation, diesel engine exhust and environmental tobacco smoke. We are now undertaking similar surveys to estimate the prevalence of occupational exposure to asthmagens, noise and ototoxic chemicals. We are also examining whether migrant workers are more likely to be exposed than the Australian born population.

Conclusions This study demonstrates a practical, web-based approach to collecting population information on occupational exposure prevalence.

\section{CANCER MORBIDITY AND MORTALITY OF INORGANIC LEAD EXPOSED WORKERS IN KOREA}

${ }^{1}$ Yeon-soon Ahn, ${ }^{2}$ Min-gi Kim. 'Dongguk University Ilsan Hospital, Goyang, Republic of Korea; ${ }^{2}$ Dongguk University Gyeongju Hospital, Gyeongju, Republic of Korea

10.1136/oemed-2014-102362.138 
Objectives This study was conducted to compare the cancer incidence in inorganic lead exposed workers with the Korean general population, and to explore the relationship between cancer mortality and blood lead levels.

Method Using from the Korean annual medical surveillance for exposure to lead, a cohort comprising 74659 inorganic lead exposed workers working between January $1^{\text {st }}, 2000$ and December $31^{\text {st }}, 2004$ was compiled. This cohort was merged with the Korea National Central Cancer Registry (KNCCR) and death registry of the Korea National Statistical Office (KNSO) in order to evaluate the cancer morbidity for these workers between 2000 and 2008 .

Results There were 793 cases cancer and, the incidence of stomach cancer (SIR 1.17, 95\% CI=1.01-1.36) was found to be elevated in lead chromate workers. Excesses were observed for kidney (2.15. 1.19-3.88) and bladder cancers (2.29. 1.1494.58 ) in lead exposed workers $\geq 20$ years of job duration., kidney cancer $(2.25 .1 .21-4.18)$ in workers with $\geq 10 \mathrm{ug} / \mathrm{dl}$ of blood lead level and lung cancer in female workers with $\geq 10$ $\mathrm{ug} / \mathrm{dl}$. Workers with $\geq 40 \mathrm{ug} / \mathrm{dl}$ of blood lead levels had a significantly higher risk of overall cancer mortality (RR: 2.75; 95\% CI: 1.06-1.98) compared with workers who had less than 10 $\mathrm{ug} / \mathrm{dl}$.

Conclusions Our study showed incidence excess of lung cancer in female workers, stomach cancer in lead chromate exposed workers and a possible dose-response relationship between $\mathrm{d}$ kidney cancers and lead exposure. Also overall cancer mortality excess was observed in high lead exposed workers.

\section{RENAL DISEASE INCIDENCE AMONG 58000 MALE WORKERS WITH BLOOD LEAD MEASUREMENTS}

Kyle Steenland, Ritam Chowdhury. Rollins Sch Pub Hlth, Emory U, Atlanta, Ga, USA

\subsection{6/oemed-2014-102362.139}

Objectives To determine whether adult lead exposure is associated with incidence of end-stage renal disease (ESRD) in a cohort with measured blood leads.

Method We studied 58000 US male adults from 11 states with blood lead levels measured between 1980-2005. Most were exposed occupationally. One-third had a single blood lead test; the remainder had a median of three. Subjects were divided into five groups by highest blood lead $(0-5,6-24,25-39,40-51 \mathrm{ug} /$ dl; $16 \%, 33 \%, 34 \%$, and $17 \%$ respectively.

Results Median follow-up was 12 years; there were 302 ESRD cases. Among those with race information (31\%), the ESRD standardised incidence ratio (SIR) (US referent) was 1.08 (0.89-1.31) overall. The SIR in the highest BL category was $1.47(0.98-2.11)$, increasing to $1.56(1.02-2.29)$ for those followed $5+$ years. For the entire cohort (race imputed), the overall SIR was $0.92(0.82-1.03)$, increasing to 1.36 (0.99$1.73)$ in the highest BL category (1.43 (1.01- 1.85) with $5+$ years follow-up). RRs in internal analyses via Cox regression (entire cohort, $5+$ years follow-up) across BL categories were 1.0 (categories 1 and 2 combined), 0.92, 1.08, and 1.96 (test for trend $p=0.003)$. The effect of lead was strongest in nonwhites.

Conclusions Data were limited by lack of detailed work history and reliance on a few blood lead tests per person to estimate exposure. Data suggest current US occupational limits on blood lead levels may need to be strengthened to avoid renal disease.

\section{CANCER INCIDENCE AND MORTALITY IN AN AUSTRALIAN COHORT OF LEAD WORKERS WITH HISTORICALLY COLLECTED BLOOD LEAD DATA}

${ }^{1}$ Malcolm Sim, ${ }^{1}$ Ewan MacFarlane, 'Stella Gwini, 'Anthony Del Monaco, ${ }^{2}$ David Mclean, ${ }^{3}$ Dino Pisaniello, 'Geza Benke. ${ }^{1}$ Centre for Occupational and Environmental Health, Monash University, Melbourne, Victoria, Australia; ${ }^{2}$ Centre for Public Health Research, Massey University, Wellington, New Zealand; ${ }^{3}$ School of Population Health and Clinical Practice, University of Adelaide, Adelaide, South Australia, Australia

\subsection{6/oemed-2014-102362.140}

Objectives To measure cancer incidence and mortality in a retrospective cohort of Australian lead-exposed workers.

Method The cohort comprised male lead workers who had been participants in state government occupational blood lead surveillance programs conducted since the 1970s. Historically collected blood lead level data were accessed from surveillance records. Linkage was undertaken to the National Death Index and the Australian Cancer Database to identify causes of death and incident cancers.

Results 4114 male subjects were followed for an average of 16.2 years, giving 68172 person years. All incident cancers were lower than expected (SIR 83, 95\% CI: 73-95). The incidence of liver cancer was elevated (SIR 217, 95\% CI 103-454), as was the incidence of oesophageal cancer (SIR 240, 95\% CI: 129-447). Among those cohort members with at least one blood lead result in excess of $30 \mu \mathrm{g} / \mathrm{dL}$, oesophageal cancer incidence was elevated (SIR 755; 95\% CI 314-1813). Other cancer types were not found to occur in excess. All cause mortality was greater than expected (SMR 111; 95\% CI 101-123) based on 406 deaths. Non-malignant digestive system deaths (SMR 167; 95\% CI 110-250) and deaths from external causes (SMR 135; 95\% CI 105-174) were also elevated.

Conclusions The increase in gastrointestinal stract cancers is consistent with some previous studies of lead workers. Confounding from liefestyle factors, such as alcohol, could not be examined. It is planned to include this cohort in an international pooling study of lead exposed workers.

\section{CHALLENGES TO OCCUPATIONAL CANCER EPIDEMIOLOGY IN QATAR}

${ }^{1,2}$ Ann Olsson, ${ }^{1}$ Rachel Denholm, 'Joachim Schüz, ${ }^{1}$ Kurt Straif, ${ }^{3}$ Fiona Bonas, ${ }^{3}$ Faleh Mohamed Hussain Ali. 'International Agency for Research on Cancer, Lyon, France; ${ }^{2}$ Karolinska Institutet, Stockholm, Sweden; ${ }^{3}$ Supreme Council of Health, Doha, Qatar

\subsection{6/oemed-2014-102362.141}

Objectives Assess exposures to occupational carcinogens in Qatar

Method IARC conducted a review of environmental carcinogens (IARC Group 1 and 2A) in Qatar 2013. Information was ascertained from ministries and a survey among Qatar Petroleum associated companies

Results Major parts of the population are migrant workers; male migrant workers are primarily recruited for the construction and the oil-and gas industry, while female migrant workers mostly do domestic work. The predominant material for construction is lime stone with increasing use of gabbro containing low silica levels compared to quartz. Only small quantities of asbestos have been used. The technologies used for natural gas extraction in Qatar are mostly closed processes.

Conclusions Workers in construction and in the oil and gas sector are mainly migrant workers who remain in the country for short 